OPEN ACCESS

Edited by:

Amalia Yanni,

Harokopio University, Greece

Reviewed by:

Suman Kapila

National Dairy Research Institute

(ICAR), India

Xiaodong Xia,

Northwest A\&F University, China

*Correspondence:

Takeshi Shimosato

shimot@shinshu-u.ac.jp

tThese authors have contributed equally to this work

Specialty section

This article was submitted to Nutrition and Microbes,

a section of the journal

Frontiers in Nutrition

Received: 28 April 2021

Accepted: 27 July 2021

Published: 19 August 2021

Citation:

Ogita T, Namai F, Mikami A, Ishiguro T,

Umezawa $K$, Uyeno $Y$ and

Shimosato T (2021) A Soybean

Resistant Protein-Containing Diet Increased the Production of Reg3r

Through the Regulation of the Gut

Microbiota and Enhanced the

Intestinal Barrier Function in Mice.

Front. Nutr. 8:701466

doi: 10.3389/fnut.2021.701466

\section{A Soybean Resistant} Protein-Containing Diet Increased the Production of Reg3y Through the Regulation of the Gut Microbiota and Enhanced the Intestinal Barrier Function in Mice

\author{
Tasuku Ogita ${ }^{1 \dagger}$, Fu Namai ${ }^{1,2+}$, Ayane Mikami ${ }^{1}$, Takahiro Ishiguro ${ }^{3}, K_{\text {Koji Umezawa }}{ }^{1}$, \\ Yutaka Uyeno ${ }^{4}$ and Takeshi Shimosato ${ }^{\text {* }}$
}

'Department of Biomolecular Innovation, Institute for Biomedical Sciences, Shinshu University, Nagano, Japan, ${ }^{2}$ Department of Pathology, Graduate School of Biomedical Sciences, Nagasaki University, Nagasaki, Japan, ${ }^{3}$ Food Research Center, Asahimatsu Foods Co., Ltd., Nagano, Japan, ${ }^{4}$ Faculty of Agriculture, Shinshu University, Nagano, Japan

The maintenance of intestinal homeostasis is necessary for a good quality of life, and strengthening of the intestinal barrier function is thus an important issue. Therefore, we focused on soybean resistant protein (SRP) derived from kori-tofu (freeze-dried tofu), which is a traditional Japanese food, as a functional food component. In this study, to investigate the effect of SRP on the intestinal barrier function and intestinal microbiota, we conducted an SRP free intake experiment in mice. Results showed that ingestion of SRP decreased the serum level of lipopolysaccharide-binding protein and induced the expression of Reg3 $\gamma$, thereby improving the intestinal barrier function. In addition, SRP intake induced changes in the cecal microbiota, as observed by changes in $\beta$-diversity. In particular, in the microbiota, the up-regulation of functional gene pathways related to the bacterial invasion of epithelial cells (ko05100) was observed, suggesting that Reg3r expression was induced by the direct stimulation of epithelial cells. The results of this study suggest that SRP is a functional food component that may contribute to the maintenance of intestinal homeostasis.

Keywords: cecal microbiota, intestinal barrier, PiCRUSt, Reg $3 \gamma$, soybean resistant protein

\section{INTRODUCTION}

The gastrointestinal tract is a frontline barrier against external factors, such as food antigens and microbes. Intestinal epithelial cells (IECs), immune cells, and the microbiota are related to each other, and together, they help maintain intestinal homeostasis (1). Disruption of the intestinal system is involved in various disorders, such as obesity and inflammatory bowel disease $(2,3)$. The intestinal barrier is classified into physical and chemical barriers, and it enables intestinal homeostasis to be maintained by separating the non-self and the host (4). The physical barrier consists of mucus and the firm linking of IECs, i.e., tight junctions. In contrast, the chemical barrier consists of antimicrobial peptides (5). In particular, the regenerating islet-derived 3 (Reg3) protein, which is a C-type lectin, plays an essential role in the segregation of the microbiota 
and IECs mainly via the sterilization of Gram-positive bacteria by binding to the microbial cell surface and perforating it $(6,7)$. It is known that Reg3 $\gamma$ is induced by the interleukin (IL)-22/signal transducer and activator of transcription 3 (STAT3) pathway (8), and IL-22 is mainly secreted by the type 3 innate lymphoid cells (ILC3) stimulated by microbiota (9). In addition, Reg3 $\gamma$ can be regulated by bacterial ligands through Toll like receptor (TLR)/Myd88 signals (10).

The enhancement and/or improvement of these barrier functions are important in achieving a better quality of life, and strengthening of the intestinal barrier function through the diet is an ideal strategy as it is generally safe and cost-efficient. In this context, we focused on resistant proteins (RPs) as functional food components. RPs are protein remnants or indigestible protein complexes that have physiological functions similar to dietary fibers (11). Several studies on the functional and health attributes of RPs have been conducted, and it has been reported that the feeding of RPs to rats increased the levels of immunoglobulin $\mathrm{A}$ and mucin, which are associated with the intestinal barrier $(12,13)$. In these studies, it was also pointed out that the intestinal microbiota that metabolizes the RPs plays an important role in the functional effects of the RPs.

Freeze-dried tofu, a traditional Japanese food that is also known as kori-tofu, contains many soybean resistant proteins (SRP) due to its characteristic manufacturing process involving freezing, thawing, and drying $(14,15)$. The intake of koritofu itself or the intake of SRP from kori-tofu improved the metabolism of lipids and sugars. Takahashi and Konishi reported that the feeding of kori-tofu to rats for 3 weeks reduced the expression of genes related to lipogenesis, and resulted in a lower serum lipid level (16). In addition, Sugano et al. revealed that SRP intake decreased the serum cholesterol level in in vivo experiments in rats (17). Although there have been several studies on the effects of SRP on metabolism, no studies have examined the effects of SRP on the intestinal barrier function and the intestinal microbiota. The purpose of the present study was to investigate the effects of SRP intake on the intestinal barrier function and the cecal microbiota in mice to further determine the functionality of SRP.

\section{MATERIALS AND METHODS}

\section{Preparation of the SRP}

SRP was prepared according to the method of Nishimura et al. with minor modifications (18). Briefly, $1 \mathrm{~g}$ of pepsin (FUJIFILM Wako Pure Chemical Co., Ltd., Tokyo, Japan) was combined with $100 \mathrm{~g}$ of soy protein (Fujipro E, Fuji Oil Co., Ltd., Osaka, Japan) suspended in distilled water; the mixture was adjusted to $\mathrm{pH}$ 1.9 with $\mathrm{HCl}$, and incubated at $37^{\circ} \mathrm{C}$ for $5 \mathrm{~h}$ with shaking. Next, the $\mathrm{pH}$ was adjusted to 7.2 with $\mathrm{NaOH}$, then $3 \mathrm{~g}$ of pancreatin (FUJIFILM Wako Pure Chemical Co., Ltd.) was added to the mixture, which was incubated with shaking at $37^{\circ} \mathrm{C}$ for another $14 \mathrm{~h}$. Following this digestion, the enzyme was inactivated by heat treatment at $80^{\circ} \mathrm{C}$ for $10 \mathrm{~min}$. The $\mathrm{pH}$ was adjusted to 4.5-5.0 with $\mathrm{HCl}$, then the mixture was centrifuged at 9,000 $\times$ $g$ for $15 \mathrm{~min}$. The pellet was washed three times with distilled water, then centrifuged at $9,000 \times g$ for $15 \mathrm{~min}$, and the resulting
TABLE 1 | The composition of SRP.

\begin{tabular}{lc}
\hline Ingredients & Composition (\%) \\
\hline Moisture & 5.1 \\
Protein & 70.1 \\
Lipid & 1.1 \\
Ash & 5.2 \\
Carbohydrate & 18.5 \\
\hline
\end{tabular}

pellet was lyophilized. The SRP was incorporated into the diet as described below.

The composition of SRP was analyzed by following the standard AOAC methods with some modifications (Table 1). Briefly, the moisture content was determined by constant weight drying in an oven at $105^{\circ} \mathrm{C}$. The protein content $(\mathrm{N} \times 6.25)$ was measured by the Kjeldahl method using a Kjeldahl system (Buchi K-426 and K-350, BUCHI, Switzerland). The lipid content was measured by $n$-hexane 2-propanol mixture (3:2) extraction using a Soxhlet system (Buchi E-816, BUCHI, Switzerland). The ash content was determined by incineration in a muffle furnace at $550^{\circ} \mathrm{C}$ for $6 \mathrm{~h}$. The remaining percentage was taken to be the carbohydrate content.

\section{Mice}

Female C57BL/6 mice (5 weeks of age) were purchased from Japan SLC (Hamamatsu, Japan) and housed in standard plastic cages in a temperature-controlled $\left(24 \pm 1^{\circ} \mathrm{C}\right)$ room with a 12/12-h light/dark cycle throughout the study.

\section{SRP-Feeding Experiment}

The SRP-feeding experiment was performed according to the schedule shown in Figure 1A. Mice were acclimatized for 1 week, then randomly allocated into two groups, the SRP group or the Control (Ctr) group. Then mice were given ad libitum access to an SRP-containing diet (SRP-diet; AIN-93G + 5\% SRP, Oriental Yeast Co., Tokyo, Japan) or a control diet (AIN-93G, Oriental Yeast), respectively, for 2 weeks. The animals also had ad libitum access to tap water and were weighed every 3-5 days during the experimental period. After 2 weeks of feeding of the SRPdiet or control diet, the mice were sacrificed, and the blood and cecum were collected. The cecum was removed from each animal at necropsy by locating and excising (with scissors) the pouch while avoiding the segments connecting to the adjacent small intestine and proximal colon. The excised tissue was sliced open, and the cecal contents were placed in a tube containing RNAlater (Thermo Fisher Scientific, Waltham, MA, USA). After $24 \mathrm{~h}$ of treatment with RNAlater, the cecal contents were separated from the RNAlater by centrifugation at $8,000 \times g$ for $5 \mathrm{~min}$, then stored at $-20^{\circ} \mathrm{C}$ until DNA extraction. Following the removal of the cecal contents, the cecal tissue itself was rinsed with physiological saline to remove any remaining contents, then flash-frozen at $-80^{\circ} \mathrm{C}$ for subsequent protein isolation and western blotting. 


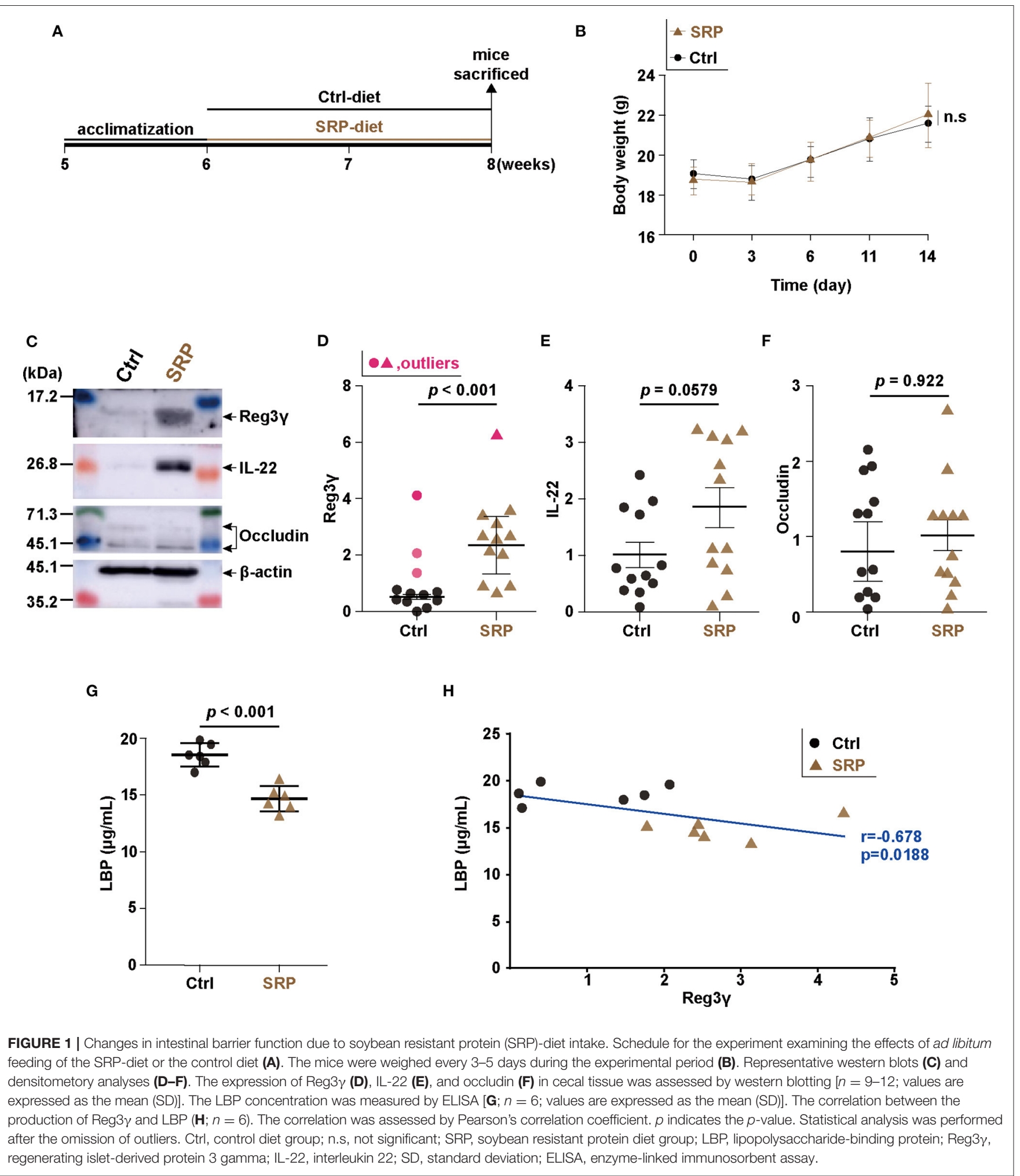

\section{Western Blotting}

The flash-frozen cecal tissue samples were suspended in lysis buffer ( $1 \%$ sodium dodecyl sulfate, $1 \%$ Triton X-100, $1 \%$ sodium deoxycholate, and $30 \mathrm{mM}$ Tris, $\mathrm{pH}$ 7.4). Then, the lysates were suspended in sample buffer (FUJFILM Wako Pure Chemical Co., Ltd.) and incubated at $95^{\circ} \mathrm{C}$ for $10 \mathrm{~min}$. Samples were resolved by $15 \%(\mathrm{v} / \mathrm{v})$ sodium dodecyl sulfate polyacrylamide gel electrophoresis. Proteins were then transferred from the gel onto 
a Hybond-P PVDF membrane (GE Healthcare Japan, Tokyo, Japan). After blocking for $1 \mathrm{~h}$, the membrane was incubated overnight with anti-mouse $\operatorname{Reg} 3 \gamma$ (SAB4301004), anti-mouse $\beta$-actin (clone AC-15), anti-mouse occludin (clone OC-3F10; Sigma-Aldrich, St. Louis, MO, USA), or anti-mouse IL-22 (clone BL35175; BioLegend, San Diego, CA, USA) antibody at $4^{\circ} \mathrm{C}$, followed by incubation with the secondary antibody (HRP Goat anti-rat IgG antibody, clone Poly4054; BioLegend). The immunoblots were visualized using ECL Prime Western Blotting Detection Reagent (GE Healthcare Japan). The protein bands were detected using ImageQuant LAS 500 (GE Healthcare Japan), and analyzed using ImageJ software (Version 1.51; National Institutes of Health, Bethesda, MD, USA). Protein quantitation was performed by densitometry; the values were normalized to the expression level of the housekeeping protein $\beta$-actin.

\section{Enzyme-Linked Immunosorbent Assay}

The collected blood was incubated for $30 \mathrm{~min}$ at $25^{\circ} \mathrm{C}$, then incubated overnight at $4^{\circ} \mathrm{C}$. The coagulated blood was removed by centrifugation $\left(1,000 \times g, 4^{\circ} \mathrm{C}, 30 \mathrm{~min}\right)$ to obtain the serum. A commercially available ELISA kit was used to quantify the lipopolysaccharide-binding protein (LBP; Biometec, Greifswald, Germany) in the serum according to the manufacturer's instructions.

\section{S rRNA V3-V4 Sequence Analysis}

An aliquot $(5-10 \mathrm{mg})$ of each cecal content sample was mixed with the Inhibitory Ex buffer $(200 \mu \mathrm{L})$ of the QIAamp Fast DNA Stool Mini Kit (Qiagen, Germantown, MD, USA) in a Zirco Prep Mini tube (Nippon Genetics Co., Ltd., Tokyo, Japan). The sample was shaken for $5 \mathrm{~min}$ using a Bug Crasher $\mu \mathrm{T}-12$ (Taitec Co., Saitama, Japan). DNA was purified from the mixture using the QIAamp Fast DNA Stool Mini Kit (Qiagen). PCR was performed using a $25-\mu \mathrm{L}$ reaction volume containing $12.5 \mu \mathrm{L}$ of $2 \times$ Gflex PCR buffer, $0.5 \mu \mathrm{L}$ of Tks Gflex DNA polymerase (TaKaRa Bio, Inc., Shiga, Japan), $1 \mu \mathrm{L}$ each of $10 \mu \mathrm{M}$ 1st PCR primers $(341 \mathrm{~F}$ and 806R, 16S rRNA gene V3-V4 regions), and $2 \mu \mathrm{L}$ of cecal DNA. PCR was performed using the following program: 1 cycle at $94^{\circ} \mathrm{C}$ for $1 \mathrm{~min}$, followed by 28 cycles at $98^{\circ} \mathrm{C}$ for $10 \mathrm{~s}, 50^{\circ} \mathrm{C}$ for $15 \mathrm{~s}$, and $68^{\circ} \mathrm{C}$ for $15 \mathrm{~s}$. Next, index PCR was performed using a $25-\mu \mathrm{L}$ reaction volume containing $12.5 \mu \mathrm{L}$ of $2 \times$ Gflex PCR buffer, $0.5 \mu \mathrm{L}$ of Tks Gflex DNA polymerase, $1 \mu \mathrm{L}$ each of Nextera XT Index primers (Illumina, Inc., San Diego, CA, USA), and $2 \mu \mathrm{L}$ of the first-round PCR product. Index PCR was performed using the following program: 1 cycle at $94^{\circ} \mathrm{C}$ for $1 \mathrm{~min}$, followed by 8 cycles at $98^{\circ} \mathrm{C}$ for $10 \mathrm{~s}, 60^{\circ} \mathrm{C}$ for $15 \mathrm{~s}$, and $68^{\circ} \mathrm{C}$ for $15 \mathrm{~s}$. After the index PCR reactions, the products were purified with the Fast Gene Gel/PCR Extraction Kit (Nippon Genetics Co., Ltd.), and quantified using Quant-iT (Life Technologies Japan, Tokyo, Japan). Equivalent quantities of PCR products from each sample were pooled and sent to TaKaRa Bio. Paired-end sequencing (250 $\mathrm{bp}$ ) of the purified PCR products was performed using MiSeq (Illumina, Inc.). The resulting sequence files, fastq files, were uploaded to DDBJ (Accession Number: PRJDB11968). Paired reads were joined by join_paired_ends using the relevant script in the QIIME 1.9.1 pipeline, an application consistent with the use of this script in similar previous studies (19). Notably, the default settings were used for "Minimum allowed overlap in base-pairs required to join pairs" and "Maximum allowed \% differences within region of overlap." The sequencing data were analyzed using the QIIME 1.9.1 pipeline, including chimera checking and the $\alpha$-/ $\beta$-diversity (19). The reads from all of the samples were clustered into operational taxonomic units (OTUs). OTU clustering was performed using the Greengenes database (gg_13_5; http://greengenes.lbl.gov/Download/) (19). We clustered sequences based on a cut-off of $97 \%$ sequence identity to the respective reference sequences. The data for species-level taxonomy were obtained by filtering the OTU tables containing taxonomic data generated by the RDP Classifier (20) at the genus level. Representative sequences were then extracted, and species-level matches within the National Center for Biotechnology Information database were identified using BLAST. The minimum percent query coverage of an alignment was $97 \%$. The setting for the alignments per read was " 1 " (default setting). The setting for the sequence similarity threshold was $97 \%$ sequencing identity (default setting). When multiple best hits with differing taxonomies were obtained, we considered both the $\mathrm{E}$ value and sequence identity. The $\alpha$-diversity index was measured using Chao1, Shannon index, phylogenetic diversity, and observed OTU analyses, while $\beta$-diversity analysis was performed using weighted and unweighted UniFrac. The characteristics of the cecal microbiota were determined as the linear discriminant analysis (LDA) score by Linear discriminant analysis Effect Size (LEfSe) version 1.0 in the Huttenhower Lab Galaxy server (21).

\section{Prediction of Cecal Microbiota Metagenomes Using PICRUSt and HUMAnN2}

Cecal microbiota metagenomes were predicted from the bacterial 16S rRNA gene by the PICRUSt (Version 1.0.0) pipeline (22). We obtained the closed-reference OTU from 16S rRNA gene data with the QIIME 1.9.1 pipeline using the Greengenes database (gg_13_5). The OTU table was normalized to the $16 \mathrm{~S}$ rRNA copy number. Metagenomes were predicted from the Kyoto Encyclopedia of Genes and Genomes database. The metagenome pathways were created by the HUMAnN2 (Version 0.7.1) pipeline (23). The characteristics of the metagenomes were determined as the LDA score from LEfSe version 1.0 in the Huttenhower Lab Galaxy server (21).

\section{Statistical Analysis}

The R software package (Version 3.3.1) was used for all statistical analyses. Outliers were identified using the Smirnov-Grubbs test and omitted prior to further statistical analysis. Normality of the variances was confirmed using the Shapiro-Wilk normality test. The statistical differences of non-normal data sets were determined by the Wilcoxon rank sum test. The F-test was used for analyzing the homogeneity of variances or homoscedasticity. The two-tailed $t$-test or Welch's $t$-test (for data with or without homoscedasticity, respectively) was used to compare the SRP group with the Ctrl group. Pearson's correlation coefficient (with 
normality) was calculated for linear and correlation analyses. A $p$-value of $<0.05$ was considered to be statistically significant.

\section{RESULTS}

\section{The SRP-Diet Induced Reg3 $\gamma$ Expression in the Cecum and Suppressed the Serum LBP Concentration}

In the SRP-diet feeding experiment, the body weight did not differ significantly between the SRP and Ctrl groups (Figure 1B). Western blotting of Reg3 $\gamma$, IL-22, and occludin from cecal tissue showed that the Reg $3 \gamma$ protein level was higher in the SRP group than in the Ctrl group (Figures 1C,D), while the levels of IL22 and occludin proteins did not differ significantly between the two groups (Figures 1C,E,F). The serum LBP concentration was significantly lower in the SRP group than in the Ctrl group (Figure 1G). In addition, the serum LBP concentration showed a significant positive correlation with the Reg $3 \gamma$ protein level in cecal tissue $(r=-0.678, p=0.0188$; Figure $1 \mathrm{H})$.

\section{The SRP-Diet Induced Changes in the Cecal Microbiota}

Considering that RPs can be assimilated by microbiota, the effect of the SRP-diet on the cecal microbiota was analyzed using a next-generation sequencing approach based on the 16S rRNA V3-V4 sequences. Diversity analysis revealed no significance changes in the $\alpha$-diversity (Chaol, phylogenetic diversity whole tree, Shannon index, and observed OTU analyses) as a result of the SRP-diet (Figures 2A-D). In contrast, principal component analysis of the $\beta$-diversity showed the formation of different clusters in the SRP and Ctrl groups, suggesting that the structure of the microbiota was changed by the SRP-diet (Figures 2E,F).

The proportions of the phyla Actinobacteria, Bacteroidetes, Firmicutes, and Proteobacteria detected did not differ between the SRP and Ctrl groups (Figures $\mathbf{3 A}, \mathbf{B}, \mathbf{D}, \mathbf{E}$ ). In contrast, the proportions of the phyla Deferribacteres and Verrucomicrobia detected were significantly lower in the SRP group than in the Ctrl group (Figures 3C,F). In addition, significant changes were observed at the species level. OTUs with a $p$-value $<0.05$, relative abundance $>1 \%$, and LDA score $>3$ in the SRP and Ctrl groups are shown in Figure 3G; among these OTUs, the 15 OTUs of the SRP group included Bacteroidetes (14 OTUs) and Firmicutes (1 OTU; Figure 3G). Representative sequences from the SRP and Ctrl groups were analyzed using BLAST to identify species-level matches within the database (Table 2).

\section{Correlation Analysis of Reg3y Expression and the OTUs}

To investigate the relationship between the microbiota and Reg $3 \gamma$ expression, we analyzed the correlation between the six bacterial OTUs that changed the most at the species level and the expression level of Reg $3 \gamma$. Results showed a significant positive correlation between the Reg $3 \gamma$ expression level and each of the six bacterial OTUs (Figure 4). Furthermore, the correlation coefficient showed that De novo 59002 (Alistipes putredinis) had a strong correlation with Reg $3 \gamma$ expression, and De novo 57882 (Flintibacter butyricus), De novo 525 (Muribaculum intestinale), De novo 18129 (M. intestinale), and De novo 52133 (M. intestinale) had a moderate correlation with Reg3 $\gamma$ expression (Figure 4).

\section{Prediction of the Functional Potential of the Microbiota in Mice Fed the SRP-Diet}

Since there is a relationship between microorganisms and their functional genes (24), we analyzed the functional attributes of the microbiome using PICRUSt. It was confirmed that the functional gene pathways involved in carbohydrate metabolism, such as other glycan degradation (ko00511), starch and sucrose metabolism (ko00500), and galactose metabolism (ko00052), were up-regulated in the SRP group when compared to the Ctrl group (Figure 5). In addition, the pathway involved in the bacterial invasion of epithelial cells (ko05100) was also up-regulated in the SRP group (Figure 5). In contrast, the functional gene pathway involved in lipopolysaccharide biosynthesis (ko00540) was down-regulated in the SRP group when compared to the Ctrl group.

\section{DISCUSSION}

In this study, we assessed the functionality of SRP derived from kori-tofu in mice with the aim of enhancing intestinal homeostasis through the use of functional food components. Free intake of feed containing SRP increased the expression of the antibacterial peptide Reg $3 \gamma$ in the cecum, suggesting that the intestinal barrier function, especially the chemical barrier, was enhanced in the mice. Indeed, the blood LBP level, a major marker of intestinal barrier failure (25-27), was significantly decreased in the SRP group. This indicated that SRP intake suppressed the invasion of microbes from the intestine into the body. Antibacterial peptides enhance the physical separation of gut bacteria from the host, which is primarily due to the bactericidal effect (28). Furthermore, Reg $3 \gamma$ is known not only for its antibacterial effect, but also for its ability to suppress apoptosis in epithelial cells (29). In fact, Reg3 $\gamma$-deficient mice have been shown to have increased contact between epithelial cells and gut bacteria, facilitating their translocation into tissues (30). These results suggest that the induction of Reg3 $\gamma$ expression by SRP strengthened the separation of the intestinal microbiota and the host, resulting in a decrease in blood LBP. In contrast, there was no change in the expression levels of IL-22, a Reg $3 \gamma$-inducing cytokine (31), and occludin, a tight junction protein (32). This suggests that $\operatorname{Reg} 3 \gamma$ is induced by pathways other than the IL-22/STAT3 pathway, and that the SRP-induced enhancement of antibacterial peptides, rather than tight junctions, plays an important role in maintaining intestinal homeostasis. However, further research is necessary to support this hypothesis because tight junction proteins other than occludin were not measured in this study and the SRP group may be divided into high and low IL-22-expressing subgroups.

Given that RPs can circumvent the digestive process and are metabolized by intestinal microbiota (11) and that the intestinal microbiota is important for Reg3 $\gamma$ expression (33), the intestinal 
A

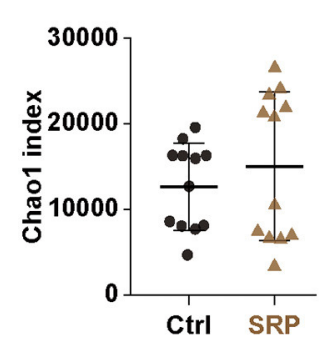

B

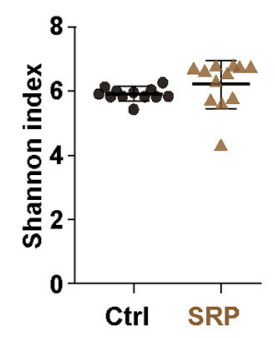

C

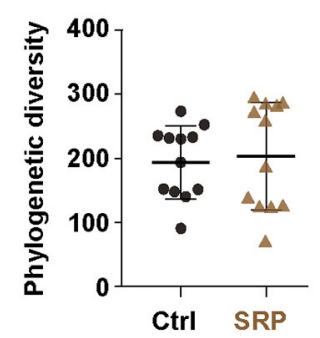

D

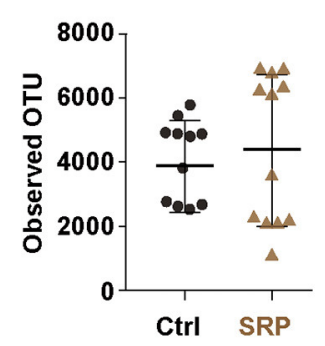

E

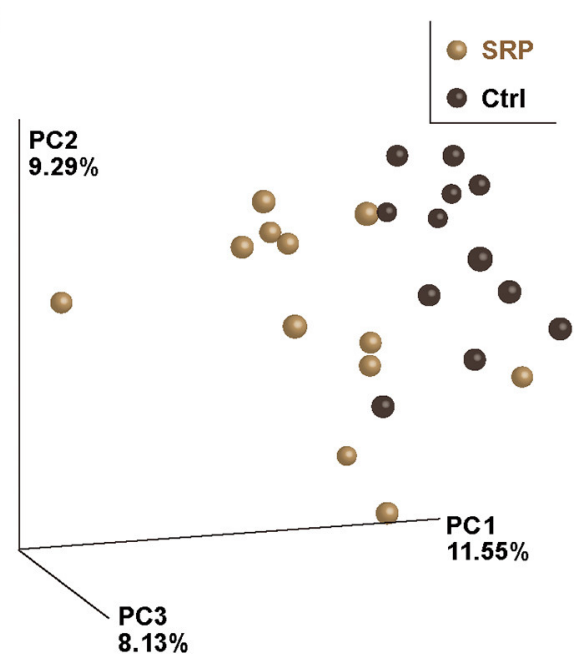

F

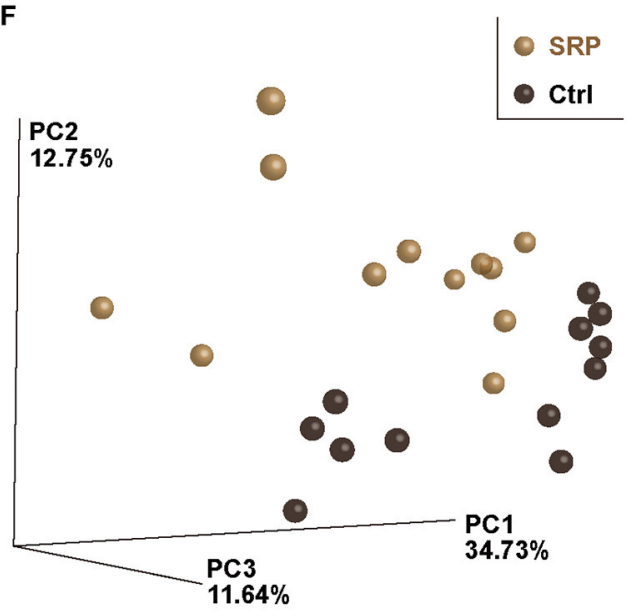

FIGURE 2 | The $\alpha$ - and $\beta$-diversity indices of the cecal microbiota. The Chao1 index (A), Shannon index (B), phylogenetic diversity (C), and observed OTUs (D) were calculated for the Ctrl and SRP groups [ $n=12$; values are expressed as the mean (SD)]. $\beta$-Diversity by weighted (E) and unweighted (F) UniFrac was assessed as the distance metric between the Ctrl and SRP groups ( $n=12)$.

microbiota was analyzed based on 16S rRNA sequence analysis. The results showed no significant change in $\alpha$-diversity, but $\beta$ diversity formed different clusters in the SRP and Crtl groups. This suggests that the ingestion of SRP changed the structure of the cecum microbiota. Indeed, the proportions of the phyla Deferribacteres and Verrucomicrobia detected were significantly lower in the SRP group than in the Ctrl group. Therefore, it was suggested that the supply of SRP to the intestinal tract influenced the microbiota. Since it was considered that the microbiota affects the expression of Reg $3 \gamma$, a correlation analysis was performed between the abundance ratio of six OTUs that differed between the SRP and Crtl groups and the expression level of Reg3 $\gamma$. Significant correlations were found for all six OTUs, and a significant correlation coefficient was obtained, especially for De novo 59002 (A. putredinis), De novo 57882 (F. butyricus), De novo 525 (M. intestinale), De novo 18129 (M. intestinale), and De novo 52133 (M. intestinale). In the future, further investigation on Reg3 $\gamma$ induction is needed using the type strain of $A$. putredinis and/or F. butyricus in a mouse model as well as in in vitro cell line experiments. Also of note, the use of enterobacteria for maintaining and improving the health of the host has been gaining much attention; this includes the use of genetically modified microorganisms, which have not been regarded as probiotics. In this context, in addition to the existing lactic acid bacteria and Bifidobacterium, a wider range of microorganisms are expected to be used in the future, and these microorganisms are referred to as nextgeneration probiotics (34). For example, it was reported that Flavonifractor plautii, a bacterium isolated from human intestine, improved inflammation $(35,36)$ and allergic immune responses (37). In addition, in recent studies, genetically modified lactic acid bacteria have been constructed to attenuate cancer (38), inflammation $(39,40)$, obesity (41), and allergy (42).

To investigate the influence of the microbiota, prediction of the functional potential of the microbiota was performed using PICRUSt. The up-regulation of pathways involved in carbohydrate breakdown was primarily observed. Actually SRP contains $18.5 \%$ of carbohydrates, but more than $70 \%$ is protein (Table 1). Since the microflora was altered for digestion of $\mathrm{SRP}$, it is necessary to further improve the purity of the protein in SRP and investigate its effect on the intestinal microbiota. It is known that Reg3 $\gamma$ expression is induced not only by the IL-22/STAT3 pathway, but also by stimulation via pattern recognition receptors, such as (33). In this context, 
A

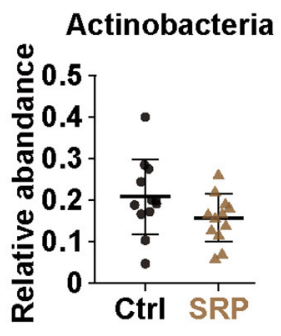

D

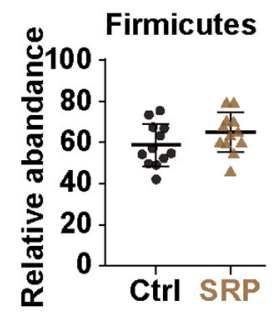

B

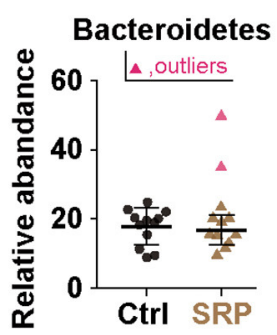

E

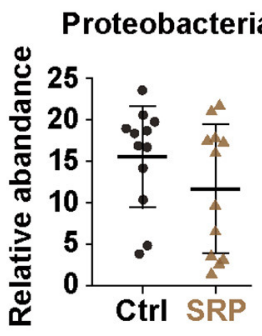

C

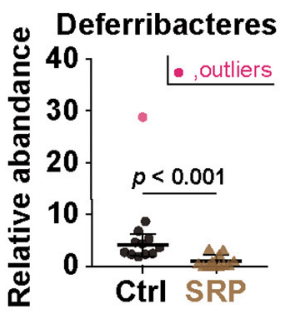

F

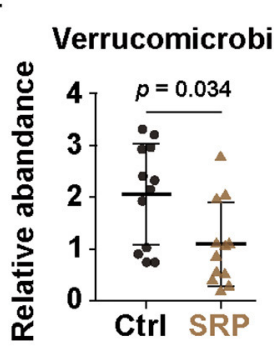

G

Ctrl $\square$ SRP

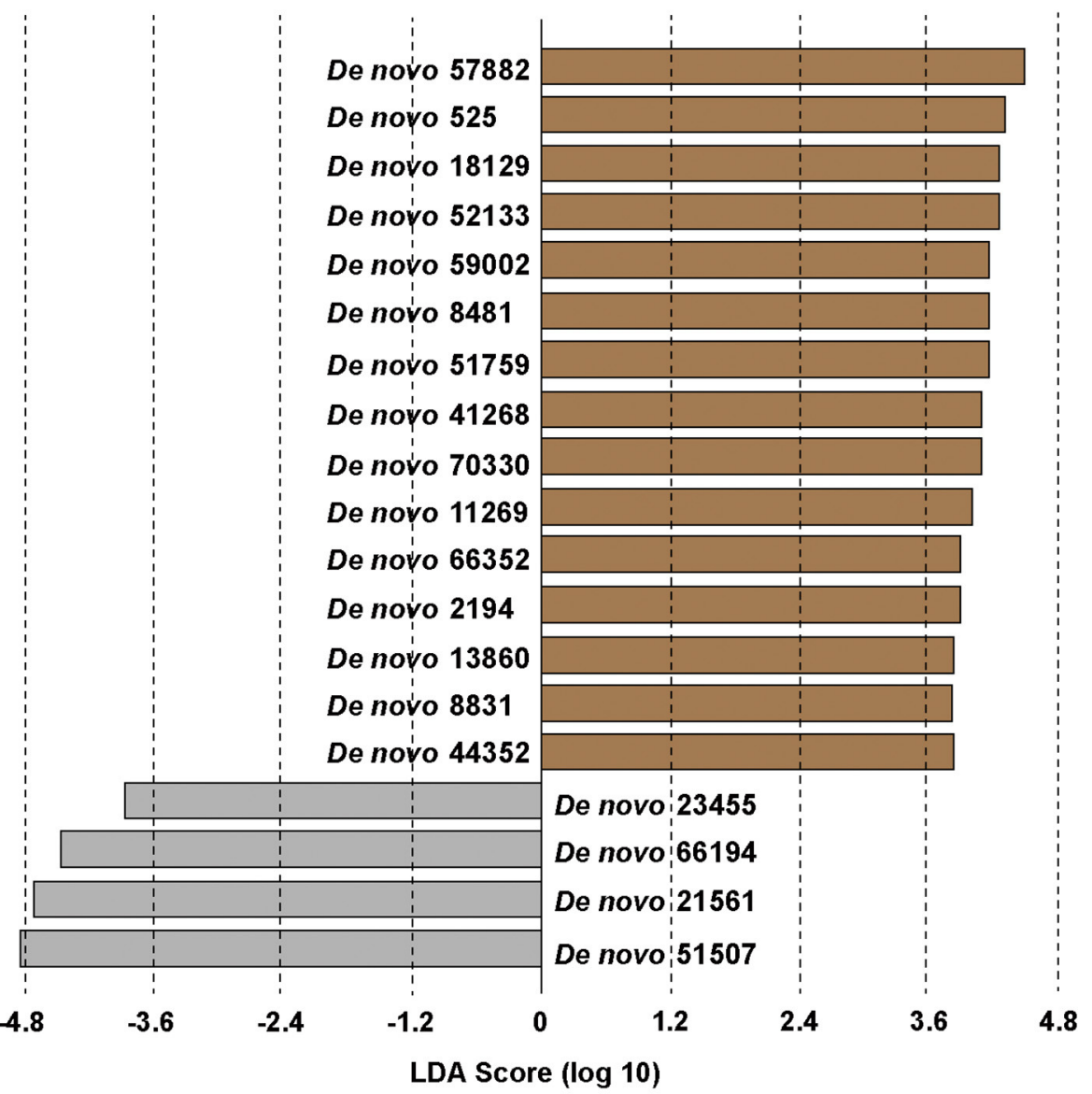

FIGURE 3 | Effects of the SRP-diet on the cecal microbiota. Proportions of the total content of Actinobacteria (A), Bacteroidetes (B), Deferribacteres (C), Firmicutes (D), Proteobacteria (E), and Verrucomicrobia (F) were assessed in the cecal contents by MiSeq $[n=10-12$; values are expressed as the mean (SD)]. $p$ indicates the $p$-value. Selection of the cecal microbiota was based on linear discriminant analysis (LDA) (G). Operational taxonomic units (OTUs) were determined to be significantly different according to the LDA score (LDA $>3)$. OTUs showing significant differences $(p<0.05)$ between the two groups were extracted by the Kruskal-Wallis and Wilcoxon rank-sum tests. Ctrl, control diet group; SRP, soybean resistant protein diet group. 
TABLE 2 | Characteristics of 19 OTUs in the cecal contents.

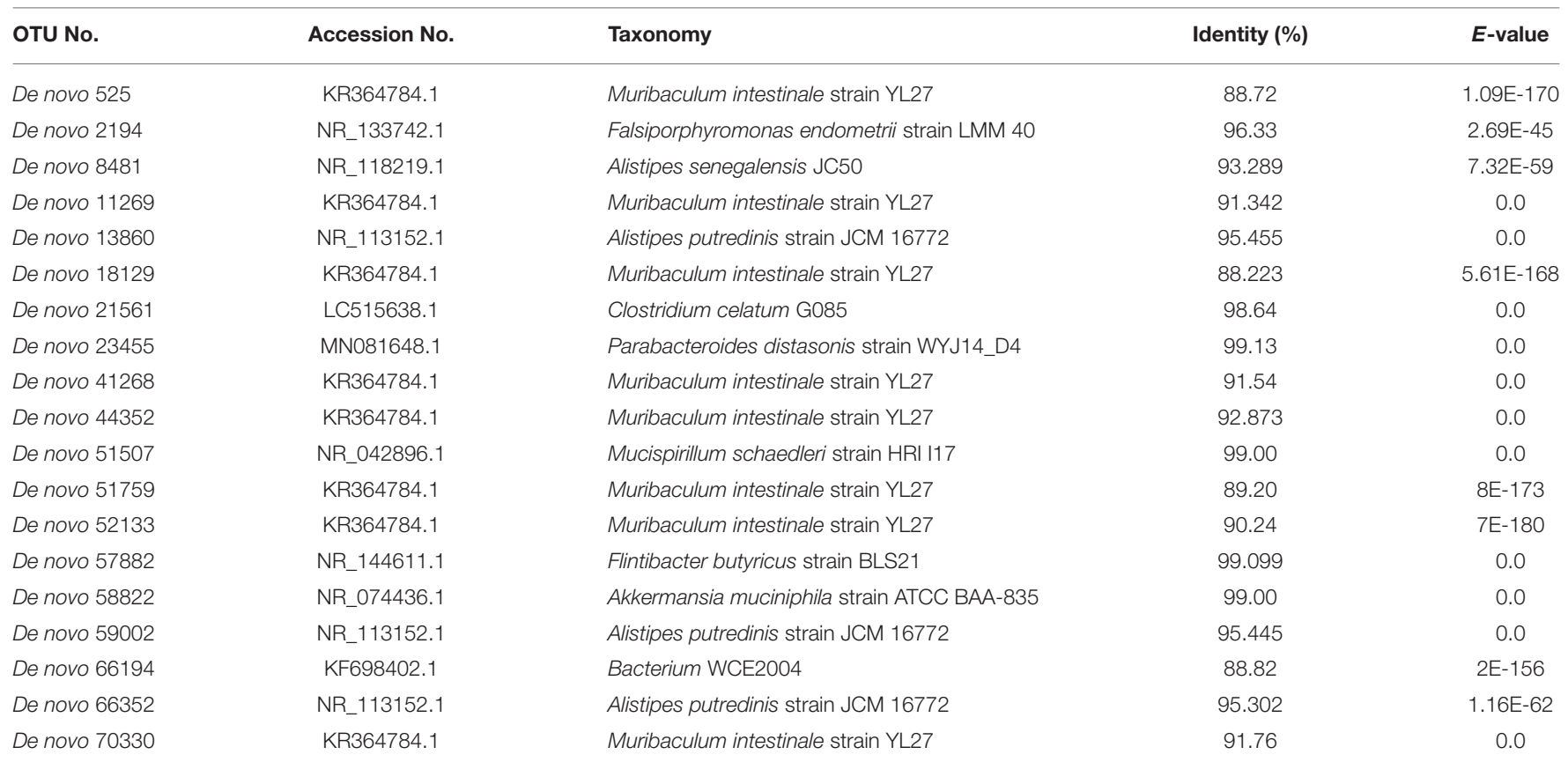

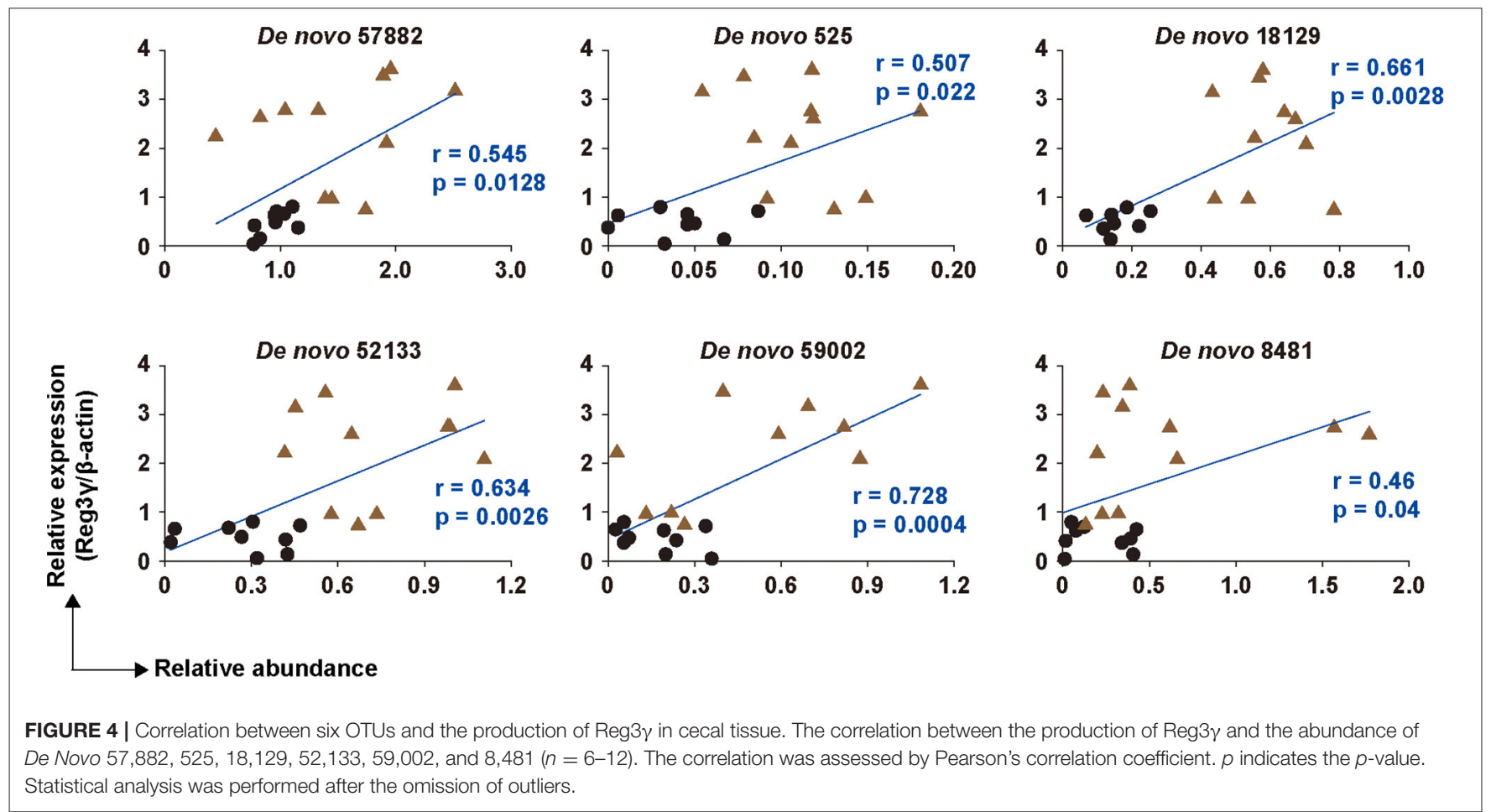

the up-regulation of the pathway involved in bacterial invasion of epithelial cells suggested that direct stimulation of IECs by the microbiota induced the increase in $\operatorname{Reg} 3 \gamma$ expression via the TLR/MyD88 pathway. Therefore, it was implied that changes in the microbiota due to SRP intake played an important role in the expression of $\operatorname{Reg} 3 \gamma$ in this study.
On the other hand, SRP intake down-regulated the pathway involved in lipopolysaccharide biosynthesis, suggesting that this pathway may be involved in the decrease of the blood LBP concentration.

In conclusion, we conducted a free intake experiment of SRP using mice with the aim of enhancing intestinal homeostasis 


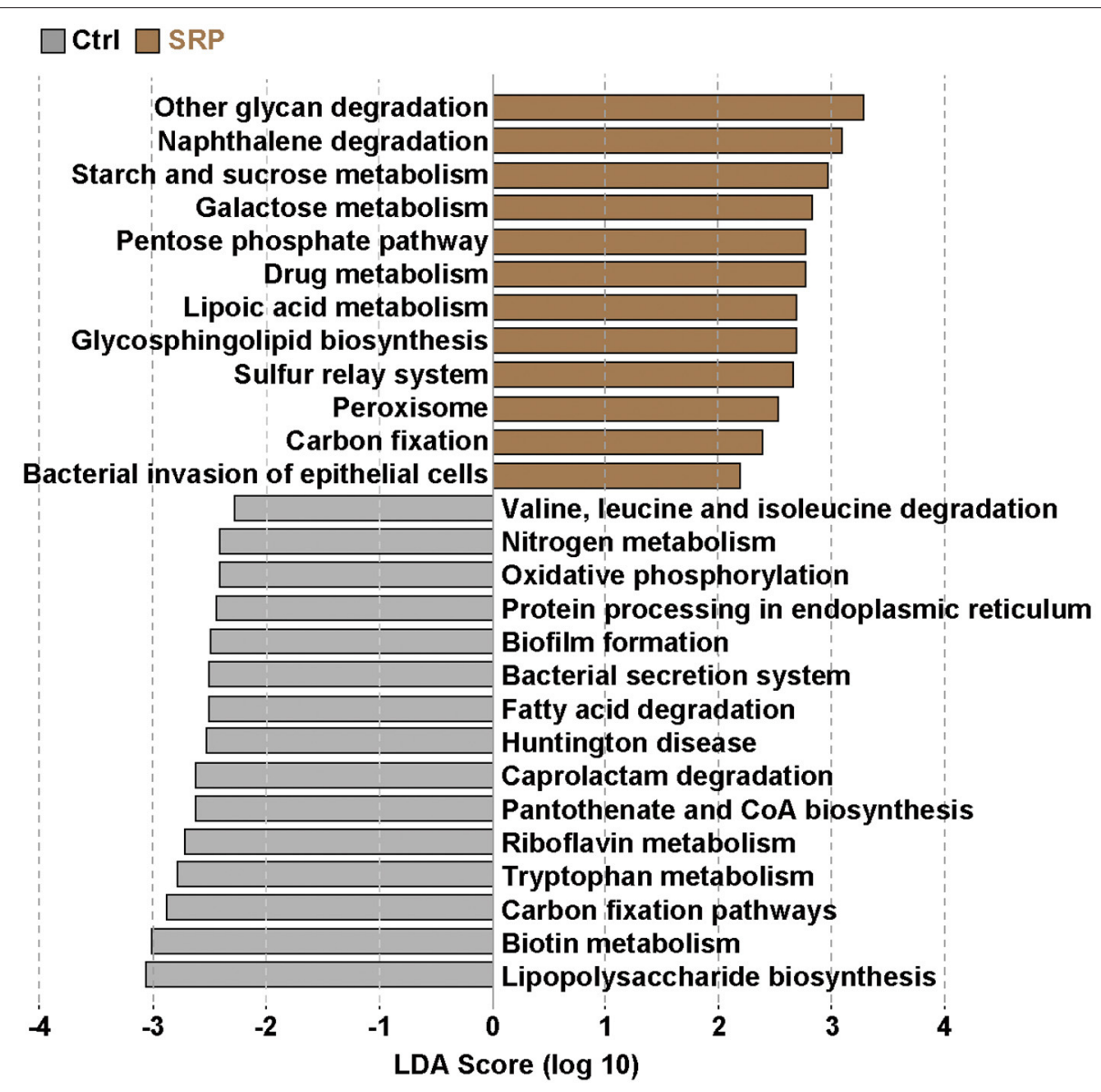

FIGURE 5 | Prediction of functional gene pathways of the cecal microbiota. The changes in the functional gene pathways in the cecal microbiota due to the SRP-diet were predicted using PICRUSt analysis. Brown bars represent the up-regulated pathways in the SRP group. In contrast, gray bars indicate the down-regulated pathways in the SRP group. Pathways were determined to be significantly different according to the LDA score (LDA > 2). Pathways showing significant differences ( $p$ $<0.05)$ between the two groups were extracted by the Kruskal-Wallis and Wilcoxon rank-sum tests. Ctrl, control diet group; SRP, soybean resistant protein diet group.

through the use of functional food components. Ingestion of SRP improved the intestinal barrier function, as seen by a decrease in the blood LBP level, and significantly promoted the expression of Reg3 $\gamma$, which is known to be involved in the chemical barrier. Since it is known that the intestinal microbiota is involved in the expression of Reg $3 \gamma$, we analyzed the cecal microbiota, and found a change in $\beta$-diversity. In addition, PICRUSt analysis showed that the SRP-diet induced the upregulation of the functional gene pathway related to the bacterial invasion of epithelial cells. Direct stimulation of the intestinal epithelium via the TLR/MyD88 pathway is known to induce the expression of $\operatorname{Reg} 3 \gamma$. Therefore, it was suggested that the SRP-induced change in the intestinal microbiota enhanced the intestinal barrier function. In the future, we will investigate the changes in the TLR/MyD88 pathway that result from SRP intake. We hope that SRP can help maintain intestinal homeostasis and contribute to human health.

\section{DATA AVAILABILITY STATEMENT}

The datasets presented in this study can be found in online repositories. The names of the repository/repositories and accession number(s) can be found below: https://www.ddbj.nig. ac.jp/, PRJDB11968.

\section{ETHICS STATEMENT}

The animal study was reviewed and approved by Committee for Animal Experiments of Shinshu University.

\section{AUTHOR CONTRIBUTIONS}

TO, FN, and AM performed the experiments. $\mathrm{FN}$ and $\mathrm{KU}$ analyzed the microbiota compositions in silico. YU and TI 
supplied the materials. TO, FN, and TS wrote the manuscript. TO and TS designed the research. TS supervised the work. All authors reviewed and approved the final manuscript.

\section{FUNDING}

This work was supported by a grant-in-aid to TO from the Takano Life Science Research Foundation, and by a grant-in-aid

\section{REFERENCES}

1. Turner JR. Intestinal mucosal barrier function in health and disease. Nat Rev Immunol. (2009) 9:799-809. doi: 10.1038/nri2653

2. Saad MJ, Santos A, Prada PO. Linking gut microbiota and inflammation to obesity and insulin resistance. Physiology (Bethesda). (2016) 31:28393. doi: 10.1152/physiol.00041.2015

3. Maloy KJ, Powrie F. Intestinal homeostasis and its breakdown in inflammatory bowel disease. Nature. (2011) 474:298306. doi: 10.1038/nature10208

4. Okumura R, Takeda K. Maintenance of intestinal homeostasis by mucosal barriers. Inflamm Regen. (2018) 38:5. doi: 10.1186/s41232-018-0063-Z

5. Ayabe T, Satchell DP, Wilson CL, Parks WC, Selsted ME, Ouellette AJ. Secretion of microbicidal alpha-defensins by intestinal paneth cells in response to bacteria. Nat Immunol. (2000) 1:113-8. doi: 10.1038/77783

6. Vaishnava S, Yamamoto M, Severson KM, Ruhn KA, Yu X, Koren $\mathrm{O}$, et al. The antibacterial lectin RegIIIgamma promotes the spatial segregation of microbiota and host in the intestine. Science. (2011) 334:2558. doi: 10.1126/science. 1209791

7. Mukherjee S, Zheng H, Derebe MG, Callenberg KM, Partch CL, Rollins D, et al. Antibacterial membrane attack by a pore-forming intestinal C-type lectin. Nature. (2014) 505:103-7. doi: 10.1038/nature12729

8. Sovran B, Loonen LM, Lu P, Hugenholtz F, Belzer C, Stolte EH, et al. IL22-STAT3 pathway plays a key role in the maintenance of ileal homeostasis in mice lacking secreted mucus barrier. Inflamm Bowel Dis. (2015) 21:53142. doi: 10.1097/MIB.0000000000000319

9. Sonnenberg GF, Fouser LA, Artis D. Border patrol: regulation of immunity, inflammation and tissue homeostasis at barrier surfaces by IL-22. Nat Immunol. (2011) 12:383-90. doi: 10.1038/ni.2025

10. Brandl K, Plitas G, Mihu CN, Ubeda C, Jia T, Fleisher M, et al. Vancomycinresistant enterococci exploit antibiotic-induced innate immune deficits. Nature. (2008) 455:804-7. doi: 10.1038/nature07250

11. Kato N, Iwami K. Resistant protein; its existence and function beneficial to health. J Nutr Sci Vitaminol (Tokyo). (2002) 48:1-5. doi: 10.3177/jnsv.48.1

12. Okazaki Y, Tomotake H, Tsujimoto K, Sasaki M, Kato N. Consumption of a resistant protein, sericin, elevates fecal immunoglobulin A, mucins, and cecal organic acids in rats fed a high-fat diet. J Nutr. (2011) 141:197581. doi: 10.3945/jn.111.144246

13. Morita T, Kasaoka S, Ohhashi A, Ikai M, Numasaki Y, Kiriyama S. Resistant proteins alter cecal short-chain fatty acid profiles in rats fed high amylose cornstarch. J Nutr. (1998) 128:1156-64. doi: 10.1093/jn/128.7.1156

14. Ishiguro T, Tatsunokuchi S, Mitsui N, Kayahara H, Murasawa H, Konishi $\mathrm{Y}$, et al. Cholesterol-lowering effect of kori-tofu protein and its highmolecular-weight fraction content. Biosci Biotechnol Biochem. (2011) 75:5757. doi: 10.1271/bbb.100634

15. Fukushima D. Soy proteins for foods centering around soy sauce and tofu. $J$ Am Oil Chem Soc. (1981) 58:346-54. doi: 10.1007/BF02582376

16. Takahashi Y, Konishi T. Tofu (soybean curd) lowers serum lipid levels and modulates hepatic gene expression involved in lipogenesis primarily through its protein, not isoflavone, component in rats. J Agric Food Chem. (2011) 59:8976-84. doi: 10.1021/jf201403u

17. Sugano M, Goto S, Yamada Y, Yoshida K, Hashimoto Y, Matsuo T, et al. Cholesterol-lowering activity of various undigested fractions of soybean protein in rats. J Nutr. (1990) 120:977-85. doi: 10.1093/jn/120.9.977 to YU and TO from the Fuji Foundation for Protein Research. This study was also funded by a grant from the Faculty of Agriculture, Shinshu University to TS.

\section{ACKNOWLEDGMENTS}

We thank the Research Center for Supports to Advanced Science, Shinshu University for use of their facilities.

18. Nishimura N, Mita Y, Sakurai M, Yamamoto T, Ota T. Effect of soy resistant protein on fermentation and microbiota in the large intestine. Daizu Tanpaku Kenkyu. (2007) 10:48-54.

19. Haas BJ, Gevers D, Earl AM, Feldgarden M, Ward DV, Giannoukos G, et al. Chimeric 16S rRNA sequence formation and detection in Sanger and 454-pyrosequenced PCR amplicons. Genome Res. (2011) 21:494504. doi: $10.1101 /$ gr.112730.110

20. Wang Q, Garrity GM, Tiedje JM, Cole JR. Naive Bayesian classifier for rapid assignment of rRNA sequences into the new bacterial taxonomy. Appl Environ Microbiol. (2007) 73:5261-7. doi: 10.1128/AEM.00062-07

21. Segata N, Izard J, Waldron L, Gevers D, Miropolsky L, Garrett WS, et al. Metagenomic biomarker discovery and explanation. Genome Biol. (2011) 12:R60. doi: 10.1186/gb-2011-12-6-r60

22. Langille MG, Zaneveld J, Caporaso JG, McDonald D, Knights D, Reyes JA, et al. Predictive functional profiling of microbial communities using 16S rRNA marker gene sequences. Nat Biotechnol. (2013) 31:81421. doi: $10.1038 /$ nbt. 2676

23. Abubucker S, Segata N, Goll J, Schubert AM, Izard J, Cantarel $\mathrm{BL}$, et al. Metabolic reconstruction for metagenomic data and its application to the human microbiome. PLoS Comput Biol. (2012) 8:e1002358. doi: 10.1371/journal.pcbi.1002358

24. Segata N, Huttenhower C. Toward an efficient method of identifying core genes for evolutionary and functional microbial phylogenies. PLoS ONE. (2011) 6:e24704. doi: 10.1371/journal.pone.0024704

25. George PJ, Anuradha R, Kumar NP, Kumaraswami V, Nutman TB, Babu S. Evidence of microbial translocation associated with perturbations in $\mathrm{T}$ cell and antigen-presenting cell homeostasis in hookworm infections. PLoS Negl Trop Dis. (2012) 6:e1830. doi: 10.1371/journal.pntd.0001830

26. Kavanagh K, Hsu FC, Davis AT, Kritchevsky SB, Rejeski WJ, Kim S. Biomarkers of leaky gut are related to inflammation and reduced physical function in older adults with cardiometabolic disease and mobility limitations. Geroscience. (2019) 41:923-33. doi: 10.1007/s11357-019-00112-z

27. Seethaler B, Basrai M, Neyrinck AM, Nazare JA, Walter J, Delzenne $\mathrm{NM}$, et al. Biomarkers for assessment of intestinal permeability in clinical practice. Am J Physiol Gastrointest Liver Physiol. (2021) 321:G117. doi: 10.1152/ajpgi.00113.2021

28. Mukherjee S, Hooper LV. Antimicrobial defense of the intestine. Immunity. (2015) 42:28-39. doi: 10.1016/j.immuni.2014.12.028

29. Zhao D, Kim YH, Jeong S, Greenson JK, Chaudhry MS, Hoepting M, et al. Survival signal REG3 $\alpha$ prevents crypt apoptosis to control acute gastrointestinal graft-versus-host disease. J Clin Invest. (2018) 128:49709. doi: 10.1172/JCI99261

30. Loonen LM, Stolte EH, Jaklofsky MT, Meijerink M, Dekker J, van Baarlen P, et al. REG3 $\gamma$-deficient mice have altered mucus distribution and increased mucosal inflammatory responses to the microbiota and enteric pathogens in the ileum. Mucosal Immunol. (2014) 7:939-47. doi: 10.1038/mi.2013.109

31. Zheng Y, Valdez PA, Danilenko DM, Hu Y, Sa SM, Gong Q, et al. Interleukin22 mediates early host defense against attaching and effacing bacterial pathogens. Nat Med. (2008) 14:282-9. doi: 10.1038/nm1720

32. Feldman GJ, Mullin JM, Ryan MP. Occludin: structure, function and regulation. Adv Drug Deliv Rev. (2005) 57:883917. doi: 10.1016/j.addr.2005.01.009

33. Vaishnava S, Behrendt CL, Ismail AS, Eckmann L, Hooper LV. Paneth cells directly sense gut commensals and maintain homeostasis at the 
intestinal host-microbial interface. Proc Natl Acad Sci USA. (2008) 105:2085863. doi: 10.1073/pnas. 0808723105

34. O'Toole PW, Marchesi JR, Hill C. Next-generation probiotics: the spectrum from probiotics to live biotherapeutics. Nat Microbiol. (2017) 2:17057. doi: $10.1038 /$ nmicrobiol.2017.57

35. Mikami A, Ogita T, Namai F, Shigemori S, Sato T, Shimosato T. Oral administration of Flavonifractor plautii, a bacteria increased with green tea consumption, promotes recovery from acute colitis in mice via suppression of IL-17. Front Nutr. (2020) 7:610946. doi: 10.3389/fnut.2020.610946

36. Mikami A, Ogita $T$, Namai F, Shigemori $S$, Sato $T$, Shimosato T. Oral administration of Flavonifractor plautii attenuates inflammatory responses in obese adipose tissue. Mol Biol Rep. (2020) 47:6717-25. doi: 10.1007/s11033-020-05727-6

37. Ogita T, Yamamoto Y, Mikami A, Shigemori S, Sato T, Shimosato T. Oral Administration of Flavonifractor plautii Strongly Suppresses Th2 Immune Responses in Mice. Front Immunol. (2020) 11:379. doi: 10.3389/fimmu.2020.00379

38. Namai F, Murakami A, Ueda A, Tsukagoshi M, Shigemori S, Ogita T, et al. Construction of genetically modified Lactococcus lactis producing anti-human-CTLA-4 single-chain fragment variable. Mol Biotechnol. (2020) 62:572-9. doi: 10.1007/s12033-020-00274-8

39. Namai F, Shigemori S, Ogita T, Sato T, Shimosato T. Microbial therapeutics for acute colitis based on genetically modified Lactococcus lactis hypersecreting IL-1Ra in mice. Exp Mol Med. (2020) 52:1627-36. doi: 10.1038/s12276-020-00507-5

40. Namai F, Yamamoto Y, Sato T, Ogita T, Shimosato T. Recombinant mouse calcitonin gene-related peptide secreted by Lactococcus lactis inhibits lipopolysaccharide-induced inflammatory response in macrophages. Anim Sci J. (2018) 89:1707-11. doi: 10.1111/asj.13115
41. Namai F, Shigemori S, Sudo K, Sato T, Yamamoto Y, Nigar S, et al. Recombinant mouse osteocalcin secreted by Lactococcus lactis promotes glucagon-like peptide-1 induction in STC-1 cells. Curr Microbiol. (2018) 75:92-8. doi: 10.1007/s00284-017-1354-3

42. Namai F, Shigemori S, Ogita $T$, Sato $T$, Shimosato T. Construction of genetically modified Lactococcus lactis that produces bioactive antiinterleukin-4 single-chain fragment variable. Mol Biol Rep. (2020) 47:703947. doi: 10.1007/s11033-020-05765-0

Conflict of Interest: TI was employed by company Asahimatsu Foods Co., Ltd.

The remaining authors declare that the research was conducted in the absence of any commercial or financial relationships that could be construed as a potential conflict of interest.

Publisher's Note: All claims expressed in this article are solely those of the authors and do not necessarily represent those of their affiliated organizations, or those of the publisher, the editors and the reviewers. Any product that may be evaluated in this article, or claim that may be made by its manufacturer, is not guaranteed or endorsed by the publisher.

Copyright (c) 2021 Ogita, Namai, Mikami, Ishiguro, Umezawa, Uyeno and Shimosato. This is an open-access article distributed under the terms of the Creative Commons Attribution License (CC BY). The use, distribution or reproduction in other forums is permitted, provided the original author(s) and the copyright owner(s) are credited and that the original publication in this journal is cited, in accordance with accepted academic practice. No use, distribution or reproduction is permitted which does not comply with these terms. 